\title{
The importance of direct and frequent communication
}

T his scenario addresses the standards of veterinary care at an institution with USDA registration for use of covered species and an NIH/OLAW assurance. As described, the institution received a citation for inadequate veterinary care due to lack of follow up and not prescribing or discussing the use of antibiotics after a wound repair on a pig. According to the AAALAC's position statement, "it is expected that the program of veterinary care will uphold the highest standards of care and ethics." This is required to both maintain animal welfare and achieve valid scientific data. Although it is not uncommon for a principal investigator (PI) to make the decision to euthanize based on their experimental goals and concerns for confounding variables, this situation may have been avoided with appropriate veterinary follow up. Dr. Meyers left instructions to call him if there were any concerns, however, three weeks between visits is not adequate follow up for a sutured wound that may dehisce or become infected. At minimum, Dr. Meyers should have copied the PI on communications post-evaluation and discussed the potential for wound infection and the use of antibiotics as needed. From the given information, it is not clear who was directly responsible for checking and documenting care of the pig. These details should have been clearly stated in the instructions left by Dr. Meyers so that the PI, facility manager, or animal care personnel could have checked on the pig and communicated with Dr. Meyers. The USDA Animal Welfare Act and Animal Welfare Regulations ${ }^{2}$ states that "daily observation of animals may be accomplished by someone other than the attending veterinarian provided that a mechanism for direct and frequent communication is in place so that timely and accurate information on problems of animal health, behavior, and well-being is conveyed to the attending veterinarian."

Although the Regulations do not specifically require the use of antibiotics, there was a lack of communication and veterinary follow-up. Therefore, the school should accept the citation and reevaluate their medical practices to prevent this problem from recurring. The institute should evaluate the written program of veterinary care description and discuss expectations of the attending veterinarian going forward. The ACLAM Guidelines for Adequate Veterinary Care ${ }^{3}$ mention that arrangements must be made to assure veterinary services are readily available to meet routine or emergency needs. The attending veterinarian should be prepared to make more frequent visits as necessary. Additionally, the animal care personnel, vivarium manger, and PI should be trained to recognize what is considered abnormal and when Dr. Meyers should be contacted outside of his regularly scheduled visits. This is especially essential to a program that does not have on-site veterinary staff to perform clinical rounds and assess the animal's recovery daily. Communication must be maintained between the veterinarian, the animal care personnel, and the PI to achieve the common goal: a high standard of animal welfare and good, valid scientific data. Finally, the facility should also reevaluate their current housing conditions to make necessary changes to prevent this from happening in the future ${ }^{4}$.

\section{Melanie Sailor*, Sumanth Putta and Donald Casebolt}

University of Southern California Department of Animal Resources, Los Angeles, California, USA.

*e-mail:melanie.sailor@med.usc.edu

Published online: 24 September 2018 https://doi.org/10.1038/s41684-018-0155-z

\section{References}

1. Association for the Assessment and Accreditation of Laboratory Animal Care International. The Attending Veterinarian and Veterinary Care. https://www.aaalac.org/accreditation/ positionstatements.cfm

2. United States Department of Agriculture. Animal Welfare Act and Animal Welfare Regulations. https://www.aphis.usda.gov/animal welfare/downloads/AC_BlueBook_AWA_FINAL_2017_508comp.pdf 3. American College of Laboratory Animal Medicine. Position Statement on Adequate Veterinary Care. https://www.aclam.org/ about/position-statements

4. Institute for Laboratory Animal Research. Guide for the Care and Use of Laboratory Animals. 8th edition, (National Academies Press, Washington, DC, 2011). 